\title{
Analysis of Source Influence on Guided Wave Excitation in Cylindrical Structures Using Spatial Fourier Transform Method
}

\author{
Yunfei Li, ${ }^{1}$ Jiang Xu ${ }^{1},{ }^{1}$ Qinghua $\mathrm{Li}^{2}$ and Guang Chen ${ }^{3}$ \\ ${ }^{1}$ School of Mechanical Science and Engineering, Huazhong University of Science and Technology, China \\ ${ }^{2}$ China Special Equipment Inspection and Research Institute, Building 2, Hepingjie, Chaoyang District, Beijing, China 10013 \\ ${ }^{3}$ AVIC Jonhon Optronic Technology Co., Ltd., China
}

Correspondence should be addressed to Jiang Xu; jiangxu@mail.hust.edu.cn

Received 26 October 2019; Accepted 18 January 2020; Published 1 February 2020

Academic Editor: Harith Ahmad

Copyright ( 2020 Yunfei Li et al. This is an open access article distributed under the Creative Commons Attribution License, which permits unrestricted use, distribution, and reproduction in any medium, provided the original work is properly cited.

Guided wave transducers, such as electromagnetic acoustic transducers and piezoelectric transducers, generate multimode waves at a given excitation frequency in a cylindrical structure, making it difficult to detect flaws in such structures. To accurately identify the flaws, the transducers must be well designed to suppress the nonaxisymmetric modes. Instead of using the normal mode expansion (NME) method, a spatial Fourier transform (SFT) method is proposed to analyze source influence on the guided wave excitation in a cylindrical structure. A two-dimensional SFT is performed on the spatial distribution function of the surface loading applied to the cylindrical structure. The spatial distribution function is represented in a cylindrical coordinate system. The circumferentialdirection SFT is carried out from the angular coordinate to the circumferential orders of the guided wave modes. The axialdirection SFT is carried out from the axial coordinate to the wavenumbers of the guided wave modes. The results of the twodimensional SFT represent guided wave excitation capabilities for different circumferential orders and wavenumbers. The specific surface loading conditions on the outer surface of a pipe are analyzed to predict source influence on the guided wave excitation. The results are consistent with those obtained using the NME method. Experiments corresponding to the specific surface loading conditions are carried out on a stainless steel pipe. The results confirm the effectiveness of the SFT method.

\section{Introduction}

Cylindrical structures, such as rods and pipes, are widely used in petrochemical and transportation industries. Because of the harsh working environment, these structures would deteriorate and thus pose a threat to safety. Therefore, cylindrical structures must be regularly inspected for corrosion to ensure safe operation $[1,2]$. The ultrasonic guided wave technology has been used for inspecting cylindrical structures $[3,4]$. This nondestructive testing technology is receiving much attention because of the possibility of longdistance inspection from a single probe position, unreachable area inspection, and high efficiency.

Guided waves, which travel in the axial direction of cylindrical structures, comprise longitudinal and torsional mode groups $[5,6]$. Typical guided wave transducers based on the piezoelectric effect $[7,8]$, Lorentz effect $[9,10]$, or magnetostrictive effect [11-15] are used to generate and receive guided waves from cylindrical structures. The surface excitation sources formed by these transducers generate multimode waves comprising axisymmetric and nonaxisymmetric modes at the given excitation frequency because of the limitations of the structure, size, and other parameters of the transducers. There are many nonaxisymmetric modes which are often with serious dispersion. The dispersion makes data analysis difficult for detection. However, it is advantageous for defect localization and focusing. The multimode waves make it difficult to detect flaws. To accurately identify the defects and improve the sensitivity, it is often desirable to obtain a single mode guided wave. Therefore, it is necessary to suppress specific modes when exciting guided waves. In addition to transducers with a single element, transducers with multiple elements have been used to improve the guided wave mode and frequency selection performance [16]. The physics of the generation process whereby the guided wave 
modes are introduced in the cylindrical structures must be carefully analyzed to determine the design parameters of the transducers influencing the characteristics of the generated field.

Two main theoretical methods have been proposed to evaluate the effects of excitation sources on guided wave excitation: the classical integral transform method [17-19] and the normal mode expansion (NME) method [20-22]. The integral transform methods, such as the Fourier transform, have been developed to solve partial differential equations. Pelts and Rose [23] employed this method to analyze source influence parameters affecting the generated field in an orthotropic plate. Koduru and Rose [24] used this method to analyze a transducer array to control an omnidirectional guided wave mode in plate-like structures. In this method, the parameters of the guided wave transducers affecting the generated field cannot be obtained directly. In the NME method, the fields generated in the structures due to loading are expanded in the form of an infinite series of normal modes, analogous to the expansion of a function in terms of any orthogonal (and complete) set of functions. The parameters affecting the amplitude are solved directly and expressed in terms of the physical properties of the guided wave modes and the excitation source. Ditri et al. [25] employed this method to analyze wedge- and comb-type transducers. From their opinion, the wedge-type transducer can be employed to generate the range of the phase velocities by the source which is proportional to the ratio of the wavelength to the size of the loading region. The comb transducer can excite the guided wave modes with small phase velocities over the wedge-type transducer. Zhang et al. [26] used this method to analyze magnetostrictive transducers. In the NME method, the derivation process of the general expression of unknown amplitude is complicated, and the general expression changes depending on the loading conditions.

The Fourier transform is a typical time and frequency domain transform method. It decomposes a function of time into the frequencies that make it up. When the method is extended to spatial domains, it is defined as the spatial Fourier transform (SFT). Because the propagating guided waves are sinusoidal in the time and spatial domains $[17,27]$, the SFT method can be employed to measure the multimode waves. Tamura [28] used a two-dimensional SFT method to measure reflection coefficients. Alleyne and Cawley [29] used a two-dimensional Fourier transform method to convert the received signals from the time and spatial domains to the frequency and wavenumber domains. Zhang et al. [30] used a one-dimensional SFT method to convert the received signals from the spatial domain to the circumferential order domain. The surface loading applied to the cylindrical structure can be considered as a summation over all the guided wave modes [20]. The surface loading applied using the guided wave transducers is distributed in the circumferential and axial directions. The advantage of the SFT method compared to the NME method in mode choosing during guided wave excitation is that the SFT can be carried out on the spatial domain distribution of the applied surface loading without complex analytical calculations. In addition, the spatial domain distribution of the applied surface loading exhibits

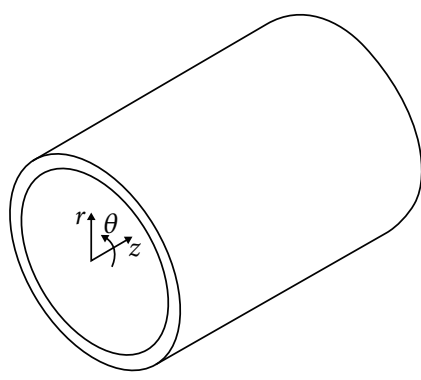

FIGURE 1: Cylindrical structure axis configuration.

periodicity in the circumferential direction and nonperiodicity in the axial direction of the cylindrical structure. Therefore, the circumferential-direction SFT can be carried out from the angular coordinate to discrete circumferential orders and the axial-direction SFT from the axial coordinate to continuous wavenumbers.

In this paper, an SFT method is proposed to analyze source influence on guided wave excitation in a cylindrical structure. The spatial distribution function of an arbitrary surface loading condition on the cylindrical structure is represented in a cylindrical coordinate system. The spatial distribution function is converted from the spatial domain to circumferential order and wavenumber domains using the two-dimensional SFT. The circumferential-direction SFT is carried out from the angular coordinate to the circumferential orders of the guided wave modes, whereas the axialdirection SFT is carried out from the axial coordinate to the wavenumbers of the guided wave modes. The results of the two-dimensional SFT represent guided wave excitation capabilities for different circumferential orders and wavenumbers. The two separated one-dimensional SFTs are performed on the separable spatial distribution functions to determine the circumferential and axial amplitude factors. Moreover, the specific surface loading conditions on the outer surface of a pipe are analyzed to predict the source influence on guided wave excitation. The results are compared with those obtained using the NME method. Finally, experiments corresponding to the specific theoretical surface loading conditions are carried out on a stainless steel pipe to test and verify the SFT method.

\section{Theoretical Background}

As shown in Figure 1, for the convenience of analysis, the central axis and radial direction of a pipe or rod are made consistent with the longitudinal and polar axes of the cylindrical coordinate system, respectively.

Generally, because the surface loading applied to the cylindrical structure can be considered as a summation over all the guided wave modes, an arbitrary surface loading condition formed by guided wave transducers on the cylindrical structure to generate guided waves can be expressed in the cylindrical coordinate system as follows:

$$
g=\sum_{n=0}^{\infty} \sum_{m=1}^{\infty}|\phi|\left|A_{m}^{n}\right| p(r, \theta, z) q(t) \vec{e}_{\xi}
$$


where $g$ denotes the total distribution function of an arbitrary surface loading condition; $p(r, \theta, z)$ and $q(t)$ denote the spatial and time-domain distribution functions, respectively; $|\Phi|$ is a constant, representing the amplitude coefficient of the pulse excitation; and $\left|A_{m}^{n}\right|$ denotes the amplitude coefficient corresponding to the different guided wave modes and is a function of the position on the dispersion curve of each guided wave mode. The integers $n$ and $m$ denote the circumferential and group orders of the guided wave modes, respectively; $\overrightarrow{e_{\xi}}(\xi=r, \theta, z)$ denotes the dominant vibration direction, corresponding to the unit coordinate vector in the cylindrical coordinate system; and $r, \theta$, and $z$ represent the radial, angular, and axial coordinates of the cylindrical coordinate system, respectively.

The elastic waves propagate in the axial direction of the cylindrical structure, with time-domain harmonic solutions. Gazis has given the expressions of the displacement components $U \xi(\xi=r, \theta, z)$ of the different guided wave modes in the cylindrical structure in the cylindrical coordinate system as follows [27]:

$$
\begin{aligned}
& U_{r}=A_{r}(r) \cos (n \theta) e^{i k z} e^{-i \omega t}, \\
& U_{\theta}=A_{\theta}(r) \sin (n \theta) e^{i k z} e^{-i \omega t}, \\
& U_{z}=A_{z}(r) \cos (n \theta) e^{i k z} e^{-i \omega t},
\end{aligned}
$$

where $A_{\xi}(r)(\xi=r, \theta, z)$ denotes the displacement amplitude composed of Bessel functions; $k=\omega / c_{p}$ denotes the wavenumbers of the guided wave modes; $c_{p}$ represents the phase velocity; $\omega=2 \pi f$ denotes the angular frequency; and $i$ denotes the imaginary unit.

As shown in Equation (3), the Fourier transform decomposes a function of time $f(t)$ into frequencies that compose it. If the original function is a function of the spatial coordinate $f(x)$, the SFT can be defined as in Equation (4):

$$
\begin{gathered}
F(\omega)=\int_{-\infty}^{+\infty} f(t) e^{-i \omega t} d t, \\
H(\tau)=\int_{-\infty}^{+\infty} f(x) e^{-i \tau x} d x,
\end{gathered}
$$

where $F(\omega)$ and $H(\tau)$ denote the Fourier transform of $f(t)$ and $f(x) ; x$ denotes the spatial coordinate; $\tau$ denotes the corresponding domain after the SFT.

The propagating guided waves in the cylindrical structure are sinusoidal in both the time and spatial domains, as shown in Equation (2). Generally, a temporal Fourier transform of the time-domain distribution function can be carried out from the time domain to the frequency domain. Therefore, a two-dimensional SFT of the spatial distribution function can be carried out from spatial to circumferential order and wavenumber domains. In the cylindrical coordinate system, the circumferential-direction SFT can be carried out from the angular coordinate to the circumferential orders, and the axial-direction SFT can be carried out from the axial coordinate to the wavenumbers. The results of the two- dimensional SFT represent guided wave excitation capabilities for different circumferential orders and wavenumbers.

The following equation is obtained by performing the SFT on the spatial distribution function of Equation (1):

$$
H(n, k)=\int_{-\infty}^{+\infty} \int_{-\infty}^{+\infty} p(\theta, z) e^{-i(n \theta+k z)} d \theta d z,
$$

where $H(n, k)$ denotes guided wave excitation capabilities for different circumferential orders and wavenumbers of the guided wave modes.

Because the unrolled, infinitely long cylindrical structure exhibits periodicity in the circumferential direction, the spatial distribution function is a periodic function with a period of $2 \pi$ under the angular coordinate, as shown in the following equation:

$$
p(\theta, z)=p(\theta+2 \pi, z) .
$$

Therefore, the Fourier series expansion of the spatial distribution function is carried out along the angular coordinate. As such, Equation (5) can be written as follows:

$H(n, k)=\frac{1}{2 \pi} \int_{-\infty}^{+\infty}\left(\int_{\varphi}^{\varphi+2 \pi} p(\theta, z) e^{-i(n \theta+k z)} d \theta\right) d z, \quad(n=0,1,2,3, \cdots)$

where $\varphi$ denotes an arbitrary initial angle. The axisymmetric modes have the circumferential order $n=0$; the nonaxisymmetric modes have the circumferential order $n \geq 1$.

Furthermore, Ditri and Rose give the decomposition formula for $p(\theta, z)$ [20]; the spatial distribution function $p(\theta, z)$ can be represented as a product of two functions, as shown in Equation (8), one describing the angular distribution function, i.e., $p_{1}(\theta)$, and the other describing the axial distribution function, i.e., $p_{2}(z)$.

$$
p(\theta, z)=p_{1}(\theta) p_{2}(z) .
$$

The separated one-dimensional SFT can be carried out as shown in Equation (9) by substituting Equation (8) into Equation (7).

$$
\begin{aligned}
H(n, k) & =H_{1}(n) H_{2}(k), \\
H_{1}(n) & =\frac{1}{2 \pi} \int_{\varphi}^{\varphi+2 \pi} p_{1}(\theta) e^{-i n \theta} d \theta, \quad(n=0,1,2,3, \cdots), \\
H_{2}(k) & =\int_{-\infty}^{+\infty} p_{2}(z) e^{-i k z} d z,
\end{aligned}
$$

where $H_{1}(n)$ denotes the guided wave excitation capabilities for different circumferential orders and is called the circumferential amplitude factor and $\mathrm{H}_{2}(k)$ denotes the guided wave excitation capabilities for different wavenumbers and is called the axial amplitude factor. The circumferential and axial amplitude factors are related to the design parameters of the guided wave transducers. 


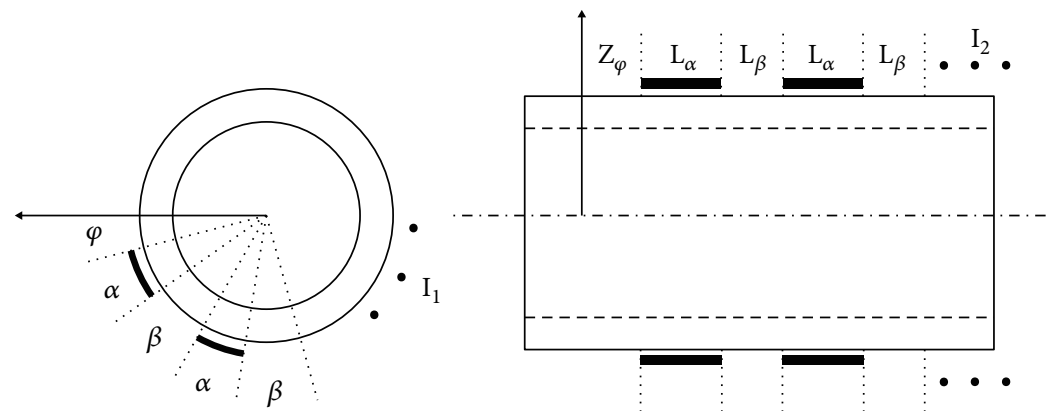

FIGURE 2: Schematic of an equally sized and spaced transducer array. $I_{1}$ and $I_{2}$ denote the number of elements in the circumferential and axial directions of the cylindrical structure, respectively; $\alpha$ denotes the angular extent of each element; $\beta=2 \pi / I_{1}-\alpha$ denotes the angular extent of each gap; $z_{\varphi}$ denotes an arbitrary initial axial position; $L_{\alpha}$ denotes the axial extent of each element; $L_{\beta}$ denotes the axial extent of each gap.

\section{Specific Loading Conditions}

The results given in the previous section are applied to a case where the loading condition is due to an equally sized and spaced transducer array, each element of which radiates a uniform pressure distribution across the outer surface of the cylindrical structure, as shown in Figure 2.

The angular distribution function in a cycle from $\varphi$ to $\varphi+2 \pi$ can be written as follows:

$p_{1}(\theta)= \begin{cases}1, & \varphi+\left(\eta_{1}-1\right) \frac{2 \pi}{I_{1}} \leq \theta \leq \varphi+\left(\eta_{1}-1\right) \frac{2 \pi}{I_{1}}+\alpha, \\ 0, & \text { otherwise, }\end{cases}$

where $\eta_{1}=1,2,3, \cdots, I_{1}$.

The axial distribution function can be written as follows:

$p_{2}(z)= \begin{cases}1, & z_{\varphi}+\left(\eta_{2}-1\right)\left(L_{\alpha}+L_{\beta}\right) \leq z \leq z_{\varphi}+\left(\eta_{2}-1\right)\left(L_{\alpha}+L_{\beta}\right)+L_{\alpha}, \\ 0, & \text { otherwise, }\end{cases}$

where $\eta_{2}=1,2,3, \cdots, I_{2}$.

3.1. Circumferential Amplitude Factor. The circumferential distribution function can be written as follows:

$H_{1}(n)= \begin{cases}\frac{1}{-2 n \pi i} e^{-i n \varphi}\left\{e^{-i n \alpha}-1\right\} \frac{1-\left(e^{-\mathrm{in}\left(2 \pi / I_{1}\right)}\right)^{I_{1}}}{1-e^{-\mathrm{in}\left(2 \pi / I_{1}\right)}} \equiv 0, & n \neq \mu I_{1}, \\ \frac{I_{1}}{-2 n \pi i} e^{-i n \varphi}\left\{e^{-i n \alpha}-1\right\}, & n=\mu I_{1},\end{cases}$

where $\mu=0,1,2,3, \cdots$.

Equation (12) shows that only modes that have a circumferential order $n$ equal to some integer $\mu$ times the number of elements $I_{1}$ in the circumferential direction of the cylindrical structure can be generated under such a loading condition. In this case, the circumferential amplitude factors of the axisymmetric guided wave modes can be determined as follows:

$$
H_{1}(0)=\frac{I_{1} \alpha}{2 \pi}
$$

This is $1 / 2 \pi$ times the total angular extent of the elements $I_{1} \alpha$ in the circumferential direction of the cylindrical structure. This result shows that the circumferential amplitude factor of the axisymmetric guided wave modes is the same for the $I_{1}$-element transducers as long as the total angular extent of the elements $\left(I_{1} \alpha\right)$ remains the same. The major difference with respect to the number of elements $I_{1}$ in the circumferential direction of the cylindrical structure is in deciding which of the higher harmonics can be generated and the relative importance of these harmonics. Generally, for a transducer array with $I_{1}$ elements in the circumferential direction of the cylindrical structure, the next family of modes that can be generated (after the axisymmetric guided wave modes) is those with a circumferential order $n$ equal to $I_{1}$, the next are those with $n=2 I_{1}$, etc. The same conclusions were obtained by Ditri and Rose [20]. The decibel scale ratio of the circumferential amplitude factor for the $\mu^{\text {th }}$ harmonics (i.e., $n=\mu I_{1}$ ) to the zero-th harmonics is defined as follows:

$$
\chi(\mu)=-20 \lg \left(\left|\frac{H_{1}\left(\mu I_{1}\right)}{H_{1}(0)}\right|\right)
$$

Figure 3 shows a plot of Equation (14) in terms of the gapto-element spacing ratio $\delta$, which is defined in

$$
\delta=\frac{\beta}{\alpha}
$$

The results are consistent with those obtained by Ditri and Rose [20]. When the gap-to-element spacing ratio $\delta$ is equal to 1 , the next family of modes with a circumferential order $n=2 I_{1}$ cannot be generated, as shown in Figure 3 . In Ditri and Rose's paper [20], they think the second generated harmonics would have an amplitude factor less than $-20 \mathrm{~dB}$ of the axially symmetric modes at the 0.66 gap-to- 


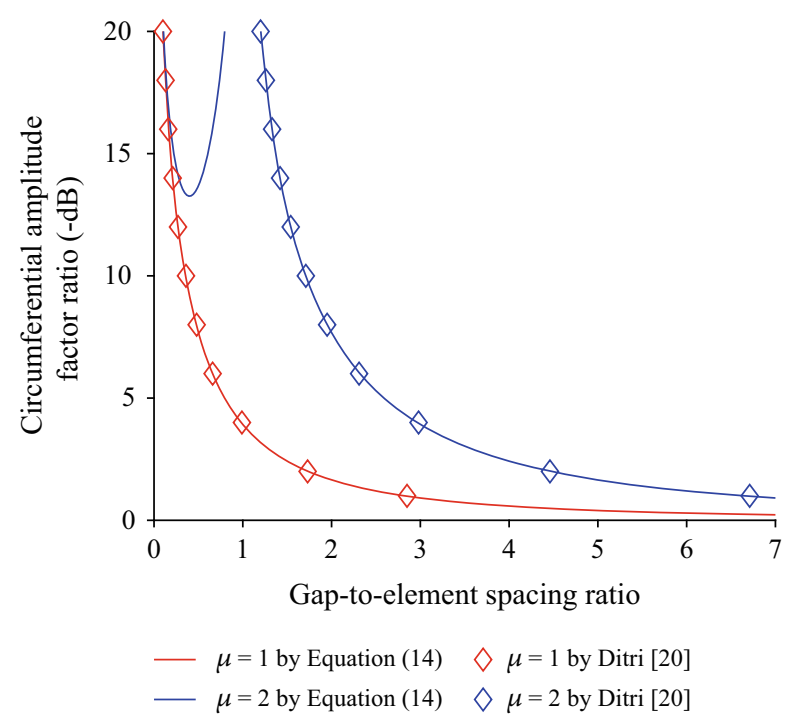

FIgURE 3: Results of circumferential amplitude factor ratio compared with those obtained using the NME method.

element ratio, which had been neglected. However, the calculated results based on NME and SFT methods as shown in Figure 3 are larger than $-20 \mathrm{~dB}$ when the gap-to-element ratio is less than 0.66 . These results mean the second harmonic modes cannot be neglected when the gap-to-element ratio is small.

3.2. Axial Amplitude Factor. The axial amplitude factors can be obtained by substituting Eq. (11) into Eq. (9) as follows.

$$
H_{2}(k)= \begin{cases}\frac{1}{-i k} e^{-i k z_{\varphi}}\left(e^{-i k L_{\alpha}}-1\right) \frac{1-\left(e^{-i k\left(L_{\alpha}+L_{\beta}\right)}\right)^{I_{2}}}{1-e^{-i k\left(L_{\alpha}+L_{\beta}\right)}}, & k \neq \frac{2 h \pi}{L_{\alpha}+L_{\beta}}, \\ \frac{I_{2}}{-i k} e^{-i k z_{\varphi}}\left(e^{-i k L_{\alpha}}-1\right), & k=\frac{2 h \pi}{L_{\alpha}+L_{\beta}},\end{cases}
$$

where $h=0,1,2,3, \cdots$.

Equation (16) goes through several maxima and minima, and the local maxima (and/or minima) occur when the wavenumbers of a given guided wave mode satisfy the following relationship:

$$
k=\frac{2 h \pi}{L_{\alpha}+L_{\beta}} .
$$

Considering the wavelength of a given guided wave mode, Equation (17) can be rewritten as follows:

$$
\lambda=\frac{L_{\alpha}+L_{\beta}}{h} .
$$

The result of the expression $L_{\alpha}+L_{\beta}$ represents the spacing between the centers of the adjacent elements in the axial direction of the cylindrical structure. Therefore, Equation

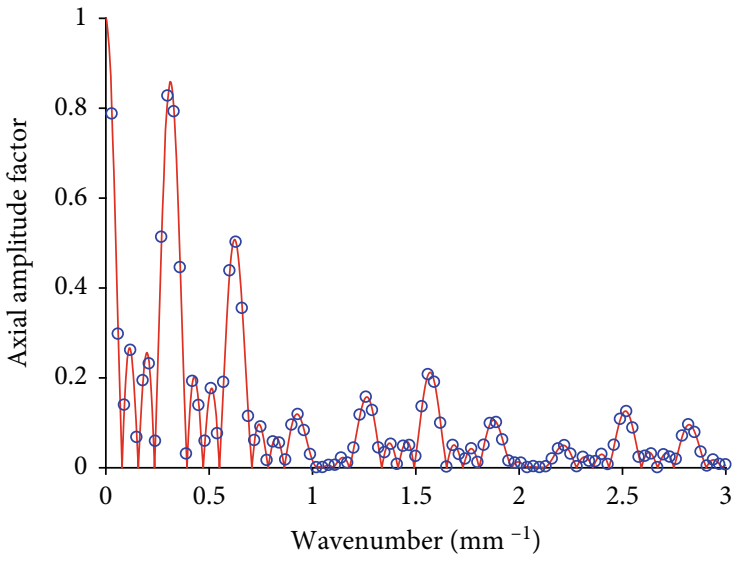

- by Equation (16)

- by Ditri [25]

FIgURE 4: Results of axial amplitude factor compared with those obtained using the NME method with $I_{2}=4, z_{\varphi}=10 \mathrm{~mm}$ (arbitrary value), $L_{\alpha}=6 \mathrm{~mm}$, and $L_{\beta}=14 \mathrm{~mm}$.

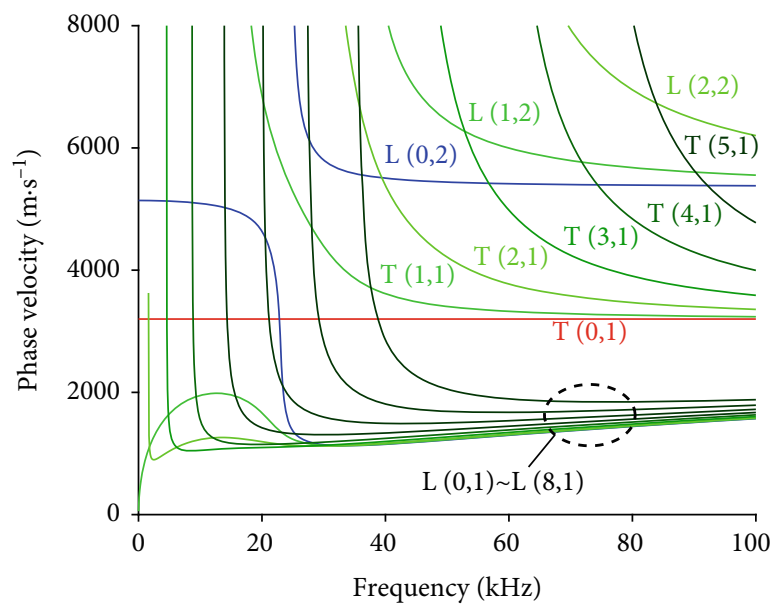

FIgURE 5: Phase velocity dispersion curve of the sample pipe.

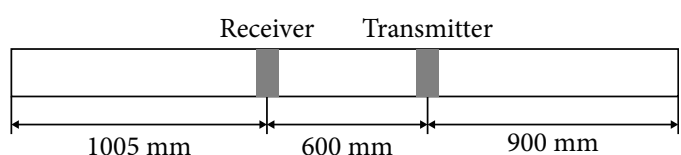

FIgURE 6: Schematic of the installation position of the transducers on the sample pipe.

(18) shows that the axial transducer array has better capabilities in generating guided wave modes which, at the given excitation frequency, have wavelengths that are equal to the spacing between the centers of the adjacent elements in the axial direction of the cylindrical structure divided by some integer. The same conclusions were drawn by Ditri et al. [25].

Figure 4 shows a normalized-scale plot of Equation (16) in terms of the wavenumbers. The results are consistent with those obtained by Ditri et al. [25]. 


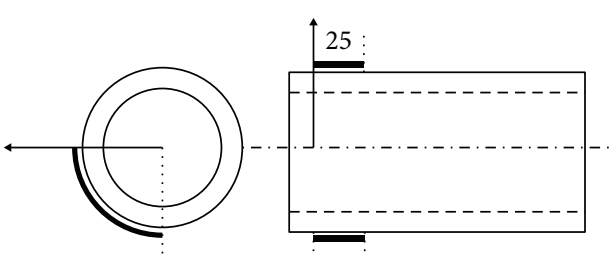

(a)

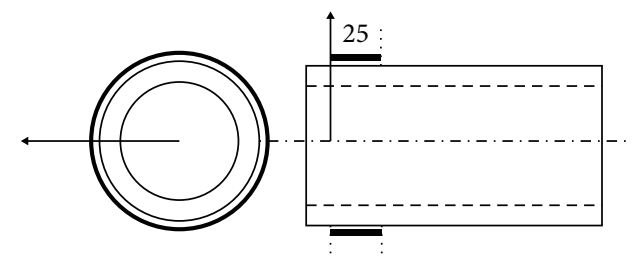

(c)
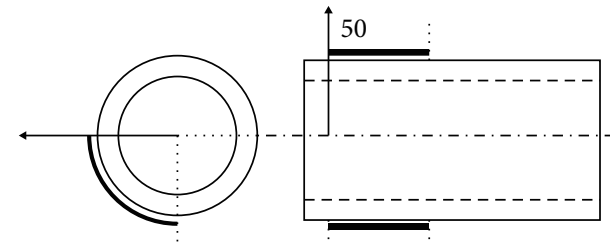

(e)

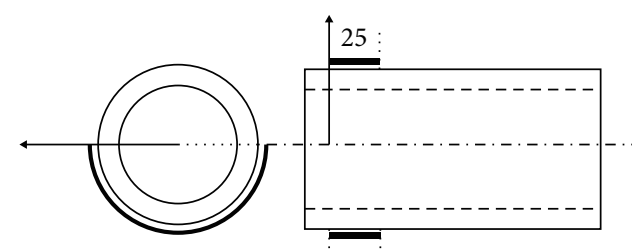

(b)

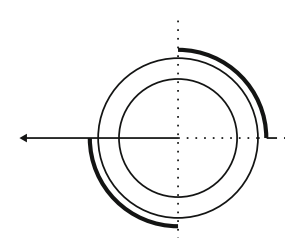

(d)
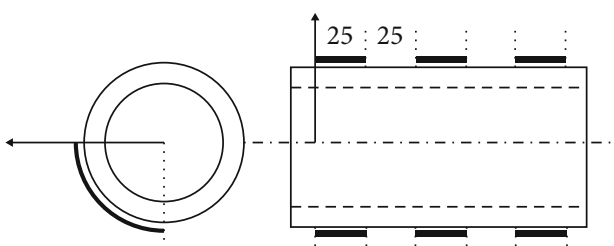

(f)

FIGURE 7: Schematics of the transmitter arrangements corresponding to the specific loading conditions. (a) No. 1 with ring coil turns: 74. (b) No. 2 with ring coil turns: 140. (c) No. 3 with ring coil turns: 280. (d) No. 4 with ring coil turns: 148. (e) No. 5 with ring coil turns: 66 (f). No. 6 with ring coil turns: 222 .

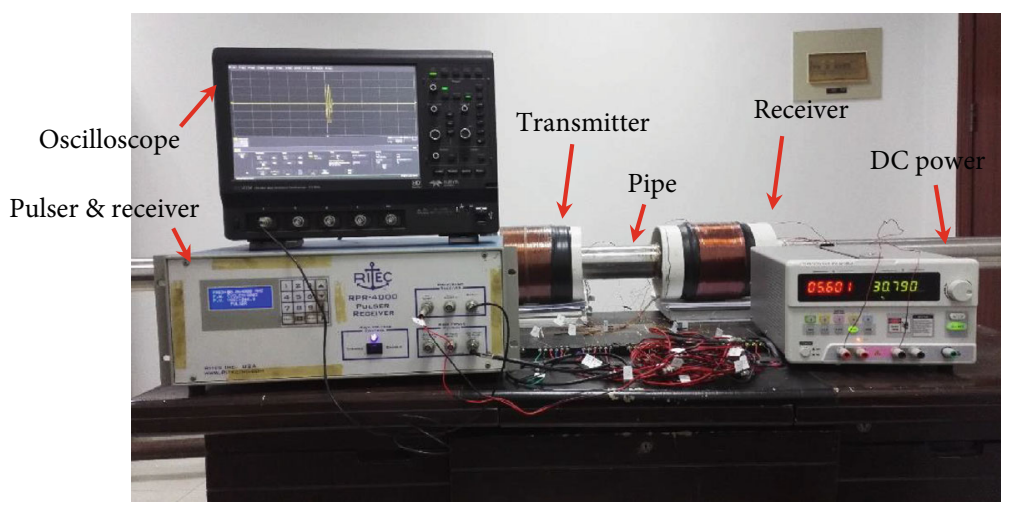

FIGURE 8: Photo of the experimental platform.

\section{Experimental Setup}

Experiments corresponding to the specific surface loading conditions were conducted to demonstrate the generation of axisymmetric and nonaxisymmetric guided wave modes in the elastic hollow cylindrical structure. The experiments were conducted on a stainless steel pipe with an outer diameter of $73 \mathrm{~mm}$, a wall thickness of $3 \mathrm{~mm}$, a length of $2505 \mathrm{~mm}$, a density of $7930 \mathrm{~kg} / \mathrm{m}^{3}$, a Young's modulus of $194 \mathrm{GPa}$, and a Poisson's ratio of 0.29 . Figure 5 shows the dispersion curves of the pipe.

The excitation signal is a three-cycle sinewave tone-burst modulated by a Hanning window. The center frequency is $64 \mathrm{kHz}$. Figure 6 shows the arrangement of the transmitter and receiver. The central distance between the transmitter and the receiver is $0.6 \mathrm{~m}$, and the center of the transmitter is $0.9 \mathrm{~m}$ from the end of the pipe. The axial length of the receiver is $25 \mathrm{~mm}$, and the receiver is divided into 16 channels in the circumferential direction to receive the displacement signals of the different angular positions and extract the nonaxisymmetric guided wave modes. Figure 7 shows the different transmitters corresponding to the specific outer surface loading conditions. The arbitrary initial angle $\varphi$ and axial position $z_{\varphi}$ are set to zero.

The cross-coiled magnetostrictive guided wave transducers [31] comprising solenoid coils, ring coils, and iron-cobalt belts were used in the experiments. The solenoid and ring coils made of enamel-insulated wires were used to provide 


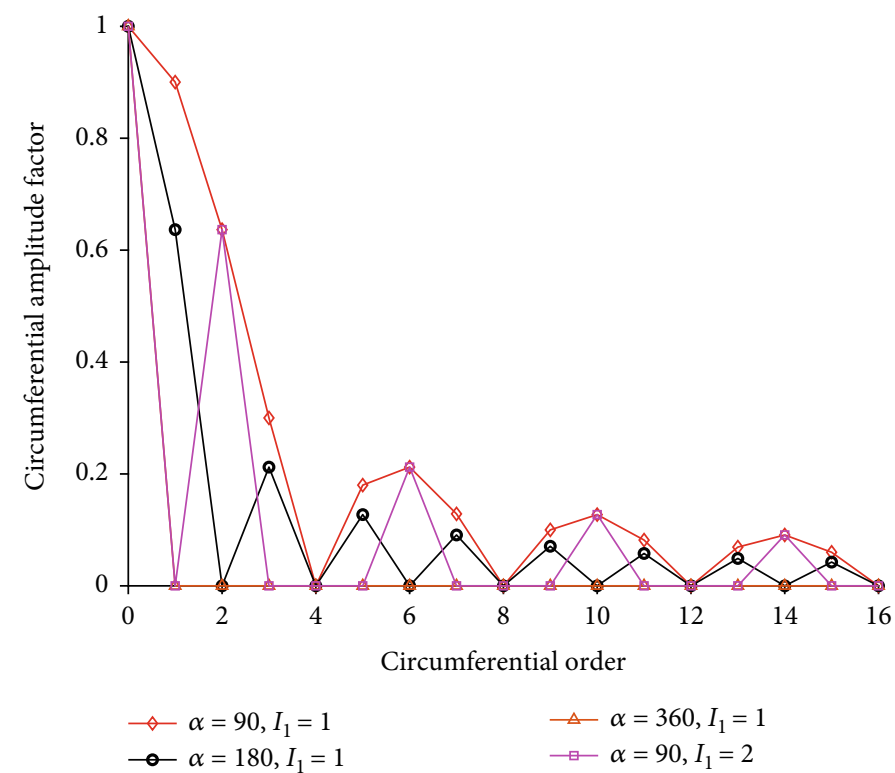

(a)

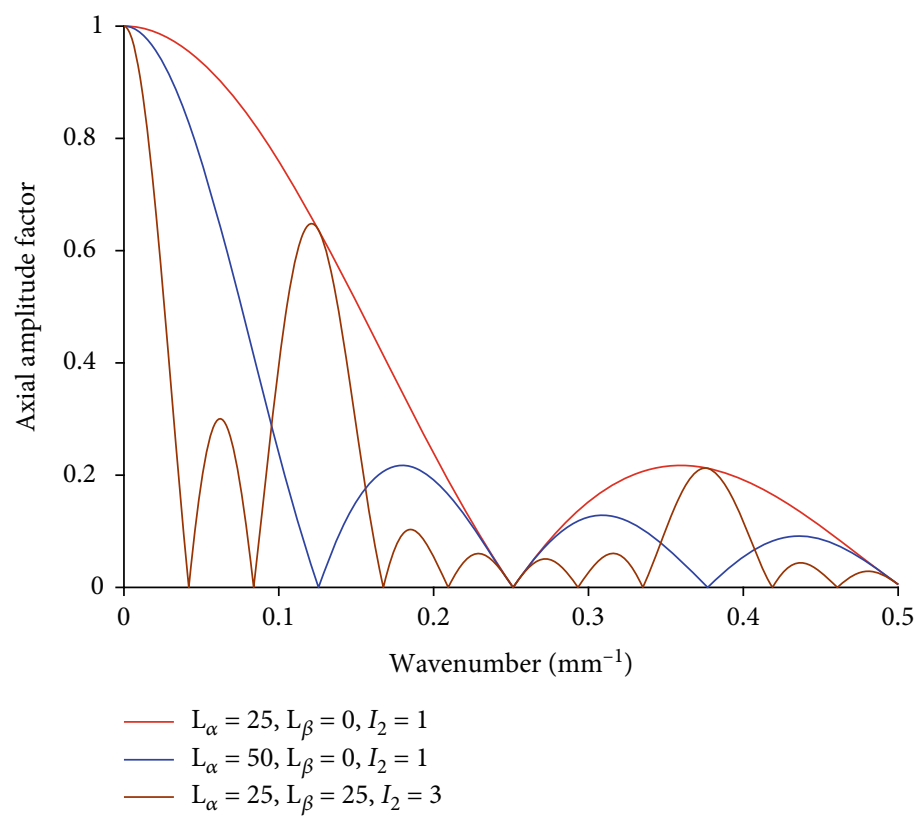

(b)

Figure 9: Normalized circumferential and axial amplitude factors corresponding to the transmitter arrangements shown in Figure 7: (a) circumferential amplitude factors; (b) axial amplitude factors.

the axial static bias magnetic field and circumferential dynamic magnetic field in the iron-cobalt belts, respectively. Based on the Wiedemann and Matteucci effects, the transducers can realize the conversion between the magnetic field signal and the circumferential displacement signal in the iron-cobalt belts. Ultrasonic vibration is transmitted between the pipe and the iron-cobalt belt via the couplant. Because of the evenly distributed (largely) static bias magnetic field and dynamic magnetic field, each element of the equally spaced transducer array is assumed to radiate a uniform pressure distribution across the outer surface of the pipe. The ironcobalt belts of the transmitters have different sizes to meet the different surface loading conditions. Each of the 16 equally spaced channels of the receiver is a 40-turn ring coil, the angular extent of which is $22.5^{\circ}$. The diameters of the enamel insulated wires of the transmitting coils and receiving coils are 0.49 and $0.21 \mathrm{~mm}$, respectively.

The RPR-4000 (manufactured by RITEC, Inc.) is employed to generate high-power excitation signals and amplify the received induced voltage signals. The DC power IPD-3012SLU (manufactured by Interlock) is employed to supply DC current to the solenoid coils. The oscilloscope HDO4034 (manufactured by Teledyne LeCroy) is employed to display, average, and store the received signals. Figure 8 


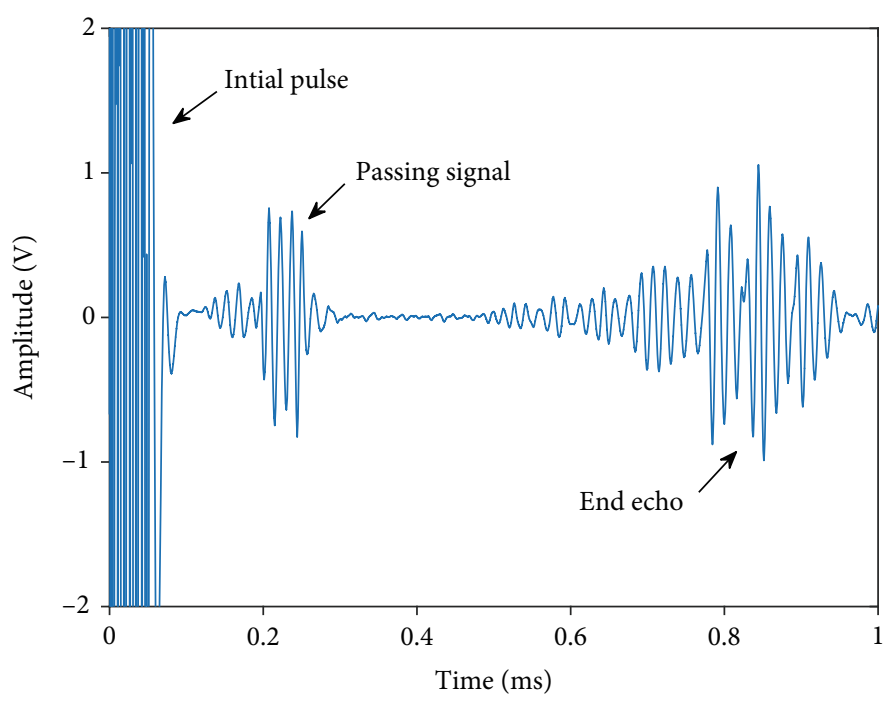

FIGURE 10: Received channel 1 signal of $\alpha=180$ and $I_{1}=1$.

shows the experimental platform used to collect the data. The peak-to-peak voltage of the excitation signal changes with the change in the transmitter; the frequency range of the bandpass filter is set to $50-800 \mathrm{kHz}$; the constant current output is set to $5.6 \mathrm{~A}$; the sampling frequency is set to $125 \mathrm{MS} / \mathrm{s}$ (megasamples per second); the received signal is averaged 50 times to reduce the white noise; and the signal amplification is set to $36.8 \mathrm{~dB}$. The induced voltage signals are amplified and recorded independently for each channel of the receiver. The total received signals from the 16 channels of the receiver form a $2 \mathrm{D}$ data matrix.

The circumferential and axial amplitude factors are calculated by substituting the parameter settings of the transmitters, shown in Figure 7, into Equations (12) and (16). Four types of angular distribution functions and three types of axial distribution functions are observed. Figures 9(a) and 9(b) show the results of the circumferential and axial amplitude factors at a normalized scale, respectively.

Overall, the wave structures of the modes in the same group are similar [17]. The torsional mode group T $(n, m)$ has dominant particle vibration in the angular direction. Therefore, at the given excitation frequency, $\mathrm{T}(n, 1)(n=0$, $1,2,3)$ is mainly considered based on the dispersion curves and wave structures. The wavelengths of $\mathrm{T}(0,1), \mathrm{T}(1,1) \mathrm{T}$ $(2,1)$, and $\mathrm{T}(3,1)$ are $50.000 \mathrm{~mm}, 51.672 \mathrm{~mm}, 57.531 \mathrm{~mm}$, and $71.984 \mathrm{~mm}$ at $64 \mathrm{kHz}$, respectively; the corresponding wave numbers are $0.126 \mathrm{~mm}^{-1}, 0.122 \mathrm{~mm}^{-1}, 0.109 \mathrm{~mm}^{-1}$, and $0.087 \mathrm{~mm}^{-1}$, respectively.

\section{Results and Discussions}

There are 16 channels in each specific loading conditions; for example, Figure 10 is the signal of experimental channel one with $\alpha=180$ and $I_{1}=1$. The focus is on the passing signal of each channel.

By adding the signals of 16 channels, a nonaxisymmetric guided wave mode with a circumferential order $n=0$ can be obtained. To extract the nonaxisymmetric guided wave modes having circumferential orders $n \geq 1$, a phase delay of $n \theta_{k} / 2 \pi$ is added to the received signal of each angular position before summation [32]. $\theta_{k}$ denotes the angular distance from the reference angular position. Figure 11 shows signals of order 0 to 2 with $\alpha=180$ and $I_{1}=1$. The maximum amplitude of the absolute value of the intercepted timedomain signal segment is used to indicate the guided wave excitation capabilities.

Figure 12 shows the experimental results at a normalized scale. As shown in Equation (2), the final displacement amplitude not only depends on the circumferential and axial amplitude factors, but also on the installation accuracy of the transducers, the sample pipe quality, the noise signals, and the uneven pressure distribution. These factors influence the final received signals and cause errors. The components of circumferential order guided waves in the signal are of primary concern to different arrangements of the transmitter.

The experimental results corresponding to the transmitter arrangements, such as in Figures $7(\mathrm{a})-7(\mathrm{c})$, show that the nonaxisymmetric modes are suppressed with the increase in the angular extent of each element. The axisymmetric modes occupy the main component when the angular extent of each element reaches $360^{\circ}$. The experimental results corresponding to Figures 7 (a) and 7(d) show that only nonaxisymmetric modes with circumferential order $n=2$ occupy the main component in addition to the axisymmetric modes because of the two-element transducer array in the circumferential direction of the pipe. These results shown in Figure 12 verify the theoretical prediction of the circumferential amplitude factor as shown in Figure 9(a).

The experimental results corresponding to Figures 7(a) and $7(\mathrm{e})$ show that nonaxisymmetric modes with circumferential order $n \geq 2$ are enhanced when the axial extent of each element reaches $50 \mathrm{~mm}$, because at the given excitation frequency, the axial amplitude factors of the $T(0,1)$ and $\mathrm{T}(1,1)$ modes are very low. The experimental results corresponding to Figures $7(\mathrm{a})$ and $7(\mathrm{f})$ show that the nonaxisymmetric modes are suppressed because at the given 


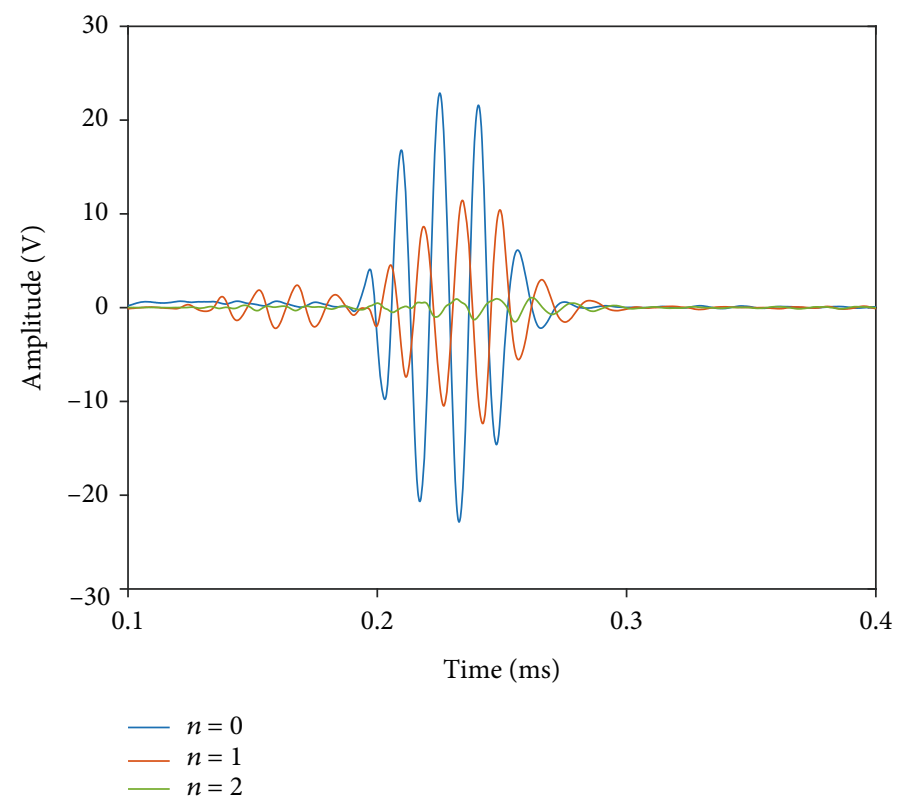

Figure 11: Passing signals of order 0 to 2 with $\alpha=180$ and $I_{1}=1$.

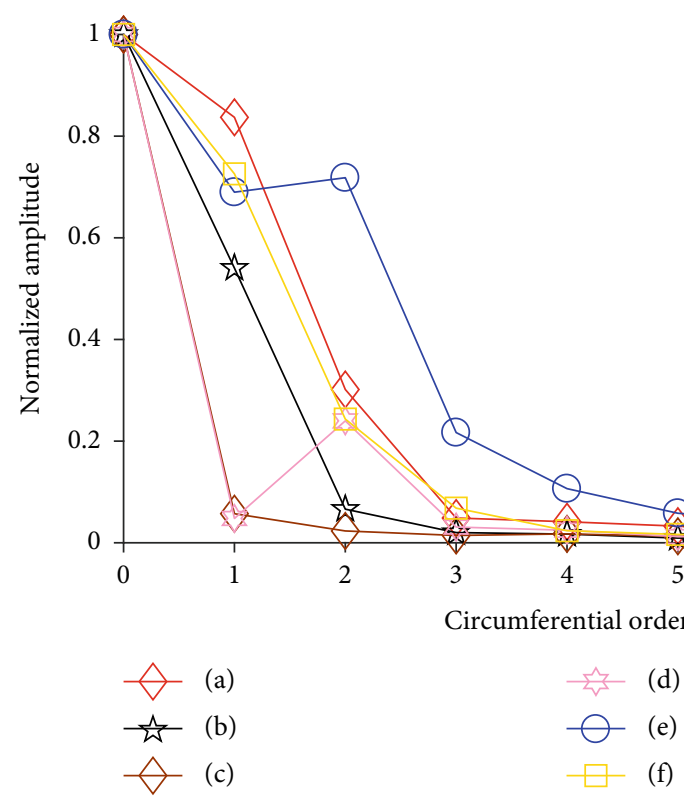

FIGURE 12: Experimental results of different transmitter arrangements (corresponding to Figure 7 ) at a normalized scale after signal processing.

excitation frequency, only the $\mathrm{T}(0,1)$ mode has a wavelength equal to the spacing between the centers of the adjacent elements of the axial transducer array; the other nonaxisymmetric modes do not. These results shown in Figure 10 verify the theoretical prediction of the axial amplitude factor as shown in Figure 9(b).

\section{Conclusions}

The proposed two-dimensional SFT method can be used to analyze source influence on guided wave excitation in cylin- drical structures. The advantage of this method is that only the spatial distribution function of an arbitrary surface loading condition on the cylindrical structure needs to be considered. The SFT method is easy to implement in practice owing to the simple algorithm. The results of the twodimensional SFT represent guided wave excitation capabilities for different circumferential orders and wavenumbers. The circumferential and axial amplitude factors can be obtained by performing the separated one-dimensional SFT on the angular and axial distribution functions, respectively. The theoretical results of the source influence on the 
guided wave excitation of specific surface loading conditions on the outer surface are consistent with those obtained using the NME method. The experimental results corresponding to the specific surface loading conditions are consistent with the theoretical predictions made using the SFT method. To suppress the nonaxisymmetric modes and enhance the axisymmetric modes, the angular extent of each transducer needs to be as close as possible to $2 \pi / I_{1} \mathrm{rad}$; the number of transducer arrays in the circumferential direction of the cylindrical structure should be greater than the maximum circumferential order of the guided wave modes at the given excitation frequency; the axial extent of each transducer and the spacing between the centers of the adjacent elements of the axial transducer array should match with the wavelengths of the axisymmetric modes at the given excitation frequency. The method provided in the paper can also be applied to guide the design process of guided wave transducers with different surface loading conditions. A time and spatial Fourier transform method which includes the timedelay function will be conducted in future works.

\section{Data Availability}

The binary data used to support the findings of this study are available from the corresponding author upon request.

\section{Conflicts of Interest}

The authors declare that they have no conflicts of interest.

\section{Acknowledgments}

This work was supported by the National Natural Science Foundation of China (Grant No. 51575213).

\section{References}

[1] M. Trokhimchuk and Y. Bakhracheva, "Accident prevention and diagnostics of underground pipeline systems," IOP Conference Series: Materials Science and Engineering, vol. 262, article 012171, 2017.

[2] S. Girgin and E. Krausmann, "Historical analysis of U.S. onshore hazardous liquid pipeline accidents triggered by natural hazards," Journal of Loss Prevention in the Process Industries, vol. 40, pp. 578-590, 2016.

[3] E. H. Ling and R. H. Abdul Rahim, "A review on ultrasonic guided wave technology," Australian Journal of Mechanical Engineering, vol. 15, pp. 1-13, 2017.

[4] Y. Y. Kim and Y. E. Kwon, "Review of magnetostrictive patch transducers and applications in ultrasonic nondestructive testing of waveguides," Ultrasonics, vol. 62, pp. 3-19, 2015.

[5] Z. Sun, L. Zhang, and J. Rose, "In Flexural longitudinal and torsional mode natural focusing phenomena in a pipe," in AIP Conference Proceedings, pp. 193-197, Green Bay, Wisconsin (USA), April 2004.

[6] Z. Sun, L. Zhang, and J. L. Rose, "Flexural torsional guided wave mechanics and focusing in pipe," Journal of Pressure Vessel Technology, vol. 127, no. 4, pp. 471-478, 2005.
[7] E. Leinov, M. J. Lowe, and P. Cawley, "Investigation of guided wave propagation and attenuation in pipe buried in sand," Journal of Sound and Vibration, vol. 347, pp. 96-114, 2015.

[8] F. Yang, L. Jing, W. Zhang, Y. Yan, and H. Ma, "Experimental and numerical studies of the oblique defects in the pipes using a chaotic oscillator based on ultrasonic guided waves," Journal of Sound and Vibration, vol. 347, pp. 218-231, 2015.

[9] N. Nakamura, H. Ogi, and M. Hirao, "EMAT pipe inspection technique using higher mode torsional guided wave $\mathrm{T}(0,2)$," NDT \& E International, vol. 87, pp. 78-84, 2017.

[10] C. Q. Gómez, F. P. García, A. Arcos, L. Cheng, M. Kogia, and M. Papelias, "Calculus of the defect severity with EMATs by analysing the attenuation curves of the guided waves," Smart Structures and Systems, vol. 19, no. 2, pp. 195-202, 2017.

[11] Z. Sun, A. Sun, and B.-F. Ju, "Guided wave imaging of oblique reflecting interfaces in pipes using common- source synthetic focusing," Journal of Sound and Vibration, vol. 420, pp. 120, 2018 .

[12] J. Xu, C. Hu, and J. Ding, “An improved longitudinal mode guided wave received sensor based on inverse magnetostrictive effect for open end pipes," Journal of Nondestructive Evaluation, vol. 38, no. 3, pp. 1-8, 2019.

[13] C. $\mathrm{Hu}$ and $\mathrm{J} . \mathrm{Xu}$, "Center frequency shift in pipe inspection using magnetostrictive guided waves," Sensors and Actuators A: Physical, vol. 298, p. 111583, 2019.

[14] J. Xu, G. Chen, J. Zhou, and Y. Li, "Effect of fatigue damage on the notch frequency of the prestressing strand based on magnetostrictive guided waves," International Journal of Applied Electromagnetics and Mechanics, vol. 59, no. 4, pp. 13411348, 2019.

[15] J. Zhou and J. Xu, "Feasibility study of fatigue damage detection of strands using magnetostrictive guided waves," International Journal of Applied Electromagnetics and Mechanics, vol. 59, no. 4, pp. 1313-1320, 2019.

[16] A. Furusawa, F. Kojima, and A. Morikawa, "Mode control of guided wave in magnetic hollow cylinder using electromagnetic acoustic transducer array," Nuclear Engineering and Technology, vol. 47, no. 2, pp. 196-203, 2015.

[17] J. L. Rose, "Ultrasonic guided waves in solid media," Cambridge University Press, pp. 247-259, 2014.

[18] C. Y. Shen, K. J. Glover, M. I. Sancer, and A. D. Varvatsis, "The discrete Fourier transform method of solving differentialintegral equations in scattering theory," IEEE Transactions on Antennas and Propagation, vol. 37, no. 8, pp. 1032-1041, 1989.

[19] E. Kuehnicke and U. Voelz, "Transient wave field calculation for ultrasonic transducers using integral transform methods," In Acoustical imaging, Springer, vol. 23, pp. 343-348, 1997.

[20] J. J. Ditri and J. L. Rose, "Excitation of guided elastic wave modes in hollow cylinders by applied surface tractions," Journal of Applied Physics, vol. 72, no. 7, pp. 2589-2597, 1992.

[21] H. J. Shin and J. L. Rose, "Guided waves by axisymmetric and non-axisymmetric surface loading on hollow cylinders," Ultrasonics, vol. 37, no. 5, pp. 355-363, 1999.

[22] J. Li and J. L. Rose, "Excitation and propagation of nonaxisymmetric guided waves in a hollow cylinder," The Journal of the Acoustical Society of America, vol. 109, no. 2, pp. 457464, 2001.

[23] S. Pelts and J. L. Rose, "Source influence parameters on elastic guided waves in an orthotropic plate," The Journal of the Acoustical Society of America, vol. 99, no. 4, pp. 2124-2129, 1996. 
[24] J. P. Koduru and J. L. Rose, "Transducer arrays for omnidirectional guided wave mode control in plate like structures," Smart Materials and Structures, vol. 22, no. 1, article 015010, 2012.

[25] J. J. Ditri, J. L. Rose, and A. Pilarski, "Generation of guided waves in hollow cylinders by wedge and comb type transducers," Review of Progress in Quantitative Nondestructive Evaluation, D. O. Thompson and D. E. Chimenti, Eds., vol. 12A, pp. 211-218, 1993.

[26] X.-W. Zhang and Z.-F. Tang, "Excitation of axisymmetric and non-axisymmetric guided waves in elastic hollow cylinders by magnetostrictive transducers," Journal of Zhejiang University Science A, vol. 17, no. 3, pp. 215-229, 2016.

[27] D. C. Gazis, “Three-dimensional investigation of the propagation of waves in hollow circular cylinders. I. Analytical foundation," The Journal of the Acoustical Society of America, vol. 31, no. 5, pp. 568-573, 1959.

[28] M. Tamura, "Spatial Fourier transform method of measuring reflection coefficients at oblique incidence. I: theory and numerical examples," The Journal of the Acoustical Society of America, vol. 88, no. 5, pp. 2259-2264, 1990.

[29] D. Alleyne and P. Cawley, "A two-dimensional Fourier transform method for the measurement of propagating multimode signals," The Journal of the Acoustical Society of America, vol. 89, no. 3, pp. 1159-1168, 1991.

[30] X. Zhang, Z. Tang, F. Lü, and X. Pan, "Excitation of dominant flexural guided waves in elastic hollow cylinders using time delay circular array transducers," Wave Motion, vol. 62, pp. 41-54, 2016.

[31] Y.-G. Kim, H.-S. Moon, K.-J. Park, and J.-K. Lee, "Generating and detecting torsional guided waves using magnetostrictive sensors of crossed coils," NDT \& E International, vol. 44, no. 2, pp. 145-151, 2011.

[32] M. Lowe, D. Alleyne, and P. Cawley, "The mode conversion of a guided wave by a part-circumferential notch in a pipe," Journal of Applied mechanics, vol. 65, pp. 649-656, 1998. 